\title{
Impacto del diseño de modelos urbanos públicos y privados sobre la respuesta térmica del sector de piedemonte. El caso del Área Metropolitana de Mendoza, Argentina
}

Ana Laura Castillo

Doctora en Ciencias, Área Energías Renovables, arquitecta. Becaria Posdoctoral en Instituto de Ambiente, Hábitat y Energía (INAHE); CONICET, Mendoza. Argentina.

alcastillo@mendoza-conicet.gob.ar

\section{Erica Correa}

Doctora en Ciencias, Área Energías Renovables. Ingeniera Química. Investigadora independiente en Instituto de Ambiente, Hábitat y Energía (INAHE); CONICET, Mendoza. Argentina. ecorrea@mendoza-conicet.gob.ar

\section{María Alicia Cantón}

DEA. Arquitecta. Investigadora principal en Instituto de Ambiente, Hábitat y Energía (INAHE); CONICET, Mendoza. Argentina.

macanton@ mendoza-conicet.gob.ar

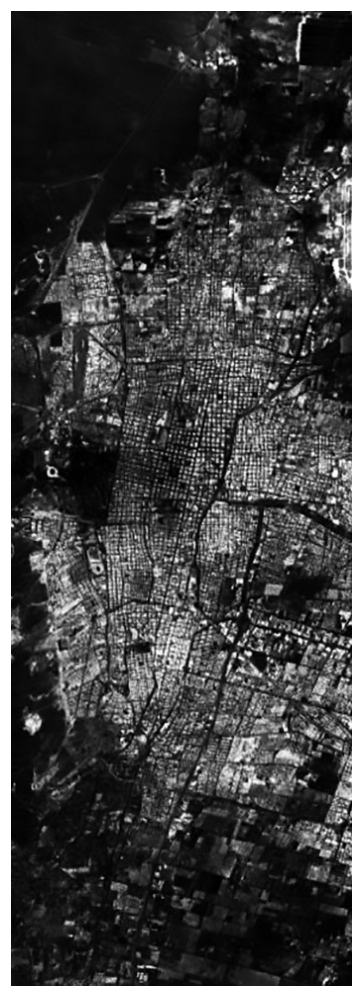

http://dx.doi.org/ 10.30972/crn.30304933 


\section{Impacto del diseño de modelos urbanos públicos y privados sobre la respuesta térmica del sector de piedemonte. El caso del Área Metropolitana de Mendoza, Argentina}

\section{Resumen}

La urbanización del piedemonte del Área Metropolitana de Mendoza ha generado una diversidad de impactos negativos sobre el territorio modificando sus condiciones naturales. Estos impactos provocaron un aumento de las superficies de absorción y retención de la radiación solar, promovieron la intensificación de la Isla de Calor Urbano (ICU) y generaron focos de ICU satélite. Este trabajo propone realizar un análisis comparativo de esquemas urbanos de origen público y privado mediante la simulación de sus comportamientos térmicos. Esto permitió identificar la relación entre el diseño de estos esquemas y su impacto en el comportamiento micro-climático del área. Los resultados obtenidos muestran que la estrategia de mayor impacto es el control de la radiación solar. En este sentido, variables de diseño como el ancho de los canales viales y la permeabilidad de los forestales, asociadas a la tipología y orientación de la trama impactan significativamente en la respuesta térmica del conjunto.

\section{Palabras clave}

Piedemonte; esquemas de urbanización; comportamiento micro-climático.

\section{Impact of the design of public and private urban models on the thermal response of the hillside sector. The case of the Metropolitan Area of Mendoza, Argentina}

\section{Abstract}

The hillside urbanization of the Mendoza Metropolitan Area has generated a wide range of negative impacts on the territory, modifying its natural conditions. These impacts caused an increase in the absorption surfaces and the retention of solar radiation, promoting the intensification of the Urban Heat Island (UHI) and generating satellite UHI focus. This work proposes to carry out a comparative analysis of urban schemes of public and private origin by simulating their thermal behavior. This allowed identifying the relationship between the design of these schemes and their impact on the microclimatic behavior of the area. The results obtained show that the strategy with the greatest impact is the control of solar radiation. In this sense, design variables such as the width of the road channels and the permeability of the forest, associated with the typology and orientation of the urban plot have a significant impact on the thermal response of the whole.

\section{Keywords}

Hillside; urbanization schemes; microclimatic behavior

\section{Impacto do desenho de modelos urbanos públicos e privados na resposta térmica do setor do piemonte. 0 caso da Área Metropolitana de Mendoza, Argentina}

Resumo

A urbanização do piemonte da Área Metropolitana de Mendoza gerou diversos impactos negativos sobre 0 território, modificando suas condições naturais. Esses impactos ocasionaram um aumento nas superfícies de absorção e retenção da radiação solar, promovendo a intensificação da Ilha de Calor Urbano (ICU) e gerando focos de ICU satélite. Este trabalho propõe-se a realizar uma análise comparativa de esquemas urbanos de origem pública e privada por meio da simulação de seu comportamento térmico. Com isso, foi possível identificar a relação entre o desenho desses esquemas e seu impacto no comportamento microclimático da área. Os resultados obtidos mostram que a estratégia de maior impacto é o controle da radiação solar. Nesse sentido, variáveis de projeto como a largura dos canais das estradas e a permeabilidade da floresta, associadas à tipologia e orientação da parcela, têm impacto significativo na resposta térmica do conjunto.

\section{Palavras chave}

Piemonte; esquemas de urbanização; comportamento microclimático. 


\section{Introducción}

Los procesos económicos, culturales y sociales internacionales, nacionales y locales han sido los causantes en la modificación y el desarrollo de las ciudades del siglo XX. El comercio y las nuevas tecnologías de movilidad y transporte generaron un crecimiento explosivo y una tendencia a la fragmentación y a la segmentación social del espacio urbano sobre la uniformidad del territorio (Romero \& Toledo, 1998; Mertins, 2000). El surgimiento y crecimiento de industrias ubicadas en la periferia hizo que se trasladara un gran número de personas hacia las ciudades. Las migraciones campo-ciudad provocaron un aumento de las dimensiones de ciudades pequeñas, lo que generó una necesidad de viviendas individuales y colectivas. Esto determinó que los esfuerzos se centraran en la planificación residencial (ZAPATERo, 2017). Debido a esta situación surge la necesidad de apropiarse del suelo y modificar su uso para la construcción de viviendas con el fin de darle solución a una demanda específica. La dimensión del suelo y su utilización siempre estuvieron fundamentalmente relacionadas con el aumento de la población, la tasa de formación de hogares y los saldos de migración, junto con una aspiración legítima de mejorar las condiciones de tamaño y calidad de las ciudades. Sin embargo, a finales del siglo XX el elevado precio del suelo del centro consolidado de las ciudades originó la expansión urbana del interior de la ciudad hacia los polígonos industriales, que modificó el uso del suelo y generó mayor demanda de los servicios (BAzANT, 2010; Giglio ET AL., 2019). Este modelo de expansión territorial de las áreas urbanas hacia las zonas periféricas se lo denomina dispersión urbana (SANcHEZ, 2015). Como consecuencia genera un elevado consumo de recursos y una diversidad de impactos negativos sobre el territorio. Este fenómeno propicia un desarrollo vertiginoso de urbanizaciones fragmentadas debido a una marcada explosión demográfica y a la rapidez de avances tecnológicos, y configura un paisaje urbano complejo (GuZMán RAMíreZ \& HeRNÁNDEZ Sainz, 2013; SzUPIANY, 2018).

En el caso de las ciudades intermedias, la presión del crecimiento urbano con patrones de asentamiento de baja densidad sobre los límites modificó el tejido de áreas cultivadas a un tejido urbano y generó además una nueva morfología y otro paisaje del territorio (HaLLER, 2017; Moschella, 2017). La expansión urbana difusa, desordenada en áreas productivas o de valor ecológico, transgrede los principios de urbanización sostenible (VILELA \& MoscheLLA, 2017, Rogers, 2001; STADel, 2000). En ese sentido, la Nueva Agenda Urbana especifica el compromiso de promover un uso sostenible de la tierra, en el cual no solo se busca evitar la 
dispersión de las nuevas áreas urbanas, sino controlar el crecimiento urbano, los cambios innecesarios del uso de suelo y disminuir la pérdida de tierras productivas y de ecosistemas frágiles (NACIONES UNIDAs, 2016).

El Gran Mendoza continuó con el modelo de ciudades intermedias internacionales, y su crecimiento siguió con los lineamientos de un desarrollo urbano expansionista. Este crecimiento urbano ha ocasionado una profunda transformación del Área Metropolitana de Mendoza (AMM) consolidando al territorio en una extensa área de tejido urbano fragmentado y heterogéneo, que dio como resultado un vasto territorio urbanizado que avanza de manera uniforme, incesante y descontrolada. Se incorporaron en la periferia focos de distinta naturaleza que estructuran la ciudad mediante un conjunto de autopistas que conectan en serie micro-ciudades continuas y funcionan como elementos organizadores del espacio-territorio (Villalobos, 2015). Este rápido crecimiento de la ciudad hizo que los límites impuestos por urbanizaciones y planes territoriales se expandieran hacia áreas cultivadas o territorios con alta fragilidad ambiental, ecológica y topográfica, como es el caso del piedemonte.

\subsection{Crecimiento urbano sobre el área de piedemonte}

El sector de piedemonte del AMM se caracteriza por ser una amplia planicie semidesértica de tipo aluvial con pendientes entre los 801 a 1400 msnm que se extienden hacia el oeste de la ciudad, al pie de la precordillera de Los Andes. En la década de 1960 comienza el proceso de crecimiento del AMM hacia los sectores de su periferia, en este caso hacia el oeste de la ciudad. Se destaca en esta década la aparición de los primeros asentamientos espontáneos y de conjuntos urbanos de origen público que ocuparon en su mayoría tierras fiscales, con alto riesgo aluvional, cerca de fallas geológicas (АвRAнам ET AL., 2005). Además, se crea el primer barrio cerrado de origen privado de la provincia, ubicado en el sector norte del área de piedemonte de la ciudad de Mendoza. Sin embargo, el crecimiento urbano hacia el piedemonte se intensifica en la década del 70. El desarrollo urbano continuó con los lineamientos de la ciudad en llanura, es decir, esquemas de trama racional, morfología urbana en forma de damero, con avenidas forestadas cuya orientación acompaña la máxima pendiente (oeste-este), que incorporan forestación no adaptada al sitio de inserción. Esto ocasionó una serie de impactos ecológicos y ambientales, ya que no se tuvieron en cuenta las características especiales que este sitio requiere. 


\section{Impacto del diseño de modelos urbanos públicos y privados \\ sobre la respuesta térmica del sector de piedemonte.}

El caso del Área Metropolitana de Mendoza, Argentina

El desarrollo de barrios privados cerrados creció de modo sostenido a partir de la década de los 80, con presencia de loteos en terrenos destinados a actividades productivas o adyacentes al área urbanizada ya consolidada. Desde el año 2004 el auge ha sido muy significativo, de acuerdo con los datos relevados por Oliva y CASTRo (2015), que indican que el $60 \%$ de los barrios surgieron a partir de ese año. En la tabla 1 se puede observar el crecimiento y expansión de los puntos que representa cada uno de los barrios privados que se asentaron sobre toda el Área Metropolitana de Mendoza. Estos conjuntos urbanos privados poseen criterios de diseño propios, lo que ocasionó un alto impacto en el terreno donde se asientan, ya que, si bien presentan un diseño urbano del tipo orgánico, para su inserción se realizaron grandes movimientos de tierra que modificaron las características naturales del terreno.

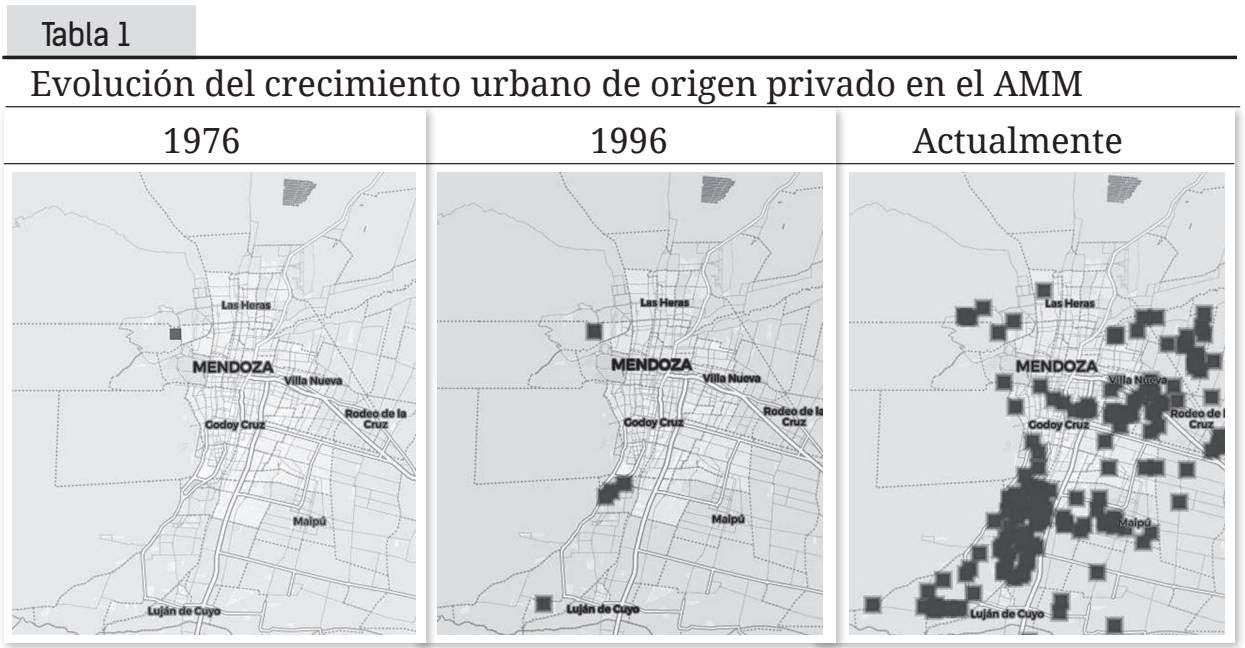

Fuente: OLIVA \& CASTRo, 2015

A lo largo de todo este proceso de crecimiento sobre el piedemonte se ha promulgado una serie de leyes y decretos: Ley 4886/83, Ley 5804/91, Decreto 1077, Ley de Ordenamiento territorial 8051; sin embargo, por las características del esquema de urbanización la legislación no tuvo resultados exitosos en el ordenamiento del crecimiento urbano sobre la configuración del territorio. Estudiado y discutido en el trabajo de Castillo et AL. (2017). 
Estas sucesivas urbanizaciones tanto públicas como privadas originaron una serie de impactos ecológicos y ambientales. Se suscitaron situaciones fragmentadas y superpuestas, en las que conviven distintas funciones no siempre compatibles entre sí (Rortman, 2003). La dispersión llevó a ocupar espacios intersticiales con serias limitantes naturales para el asentamiento humano, zonas de gran "fragilidad natural", debido a la presencia de fallas sísmicas, pendientes pronunciadas, zonas de riesgos de erosión, aluvionales, etc. (Abraham et al., 2005). Dentro de los impactos ecológicos se observa el aumento notable de los volúmenes de los escurrimientos pluviales y la disminución de los tiempos de concentración, con el consecuente aumento de caudales; esto se agravó en los últimos años por la excesiva urbanización sin criterio urbanístico en las primeras estribaciones piedemontanas (MAZA ET AL., 2004). Además, grandes movimientos de tierra afectaron considerablemente no solo el paisaje natural, tan valioso para sus habitantes, sino también la flora y fauna autóctonas. Entre los impactos ambientales se destacan la pérdida de suelo natural y su biodiversidad, el sellado de terrenos productivos y el aumento de contaminación atmosférica y sonora. Por otra parte, el crecimiento sobre el piedemonte ha dificultado los procesos de renovación y purificación del aire urbano y ha producido un aumento de las superficies de absorción y retención de la radiación solar (inercia térmica). Estos factores promovieron la intensificación de la Isla de Calor Urbano (ICU) en la ciudad y generaron focos de ICU satélite. En estudios realizados (CORREA, 2006) se determinó la existencia del fenómeno de isla de calor urbana sobre las urbanizaciones del piedemonte con una intensidad máxima de $9^{\circ} \mathrm{C}$, valor semejante al que alcanza el ICU en el sector central del AMM $\left(10^{\circ} \mathrm{C}\right)$.

Diversos estudios han permitido desarrollar una amplia variedad de estrategias para contrarrestar el impacto microclimático que genera el aumento de la temperatura, como por ejemplo el diseño y materialidad de los elementos de la envolvente urbana o a partir de la incorporación de techos verdes (GILL ET AL., 2007; FloREs, 2019), envolventes con altos niveles de albedo (Georgescu $E T$ AL., 2014), enfriamiento por pulverización de techo (NARUMI ET AL., 2009); reducción del calor antropogénico liberado (CHrysoulaKIs ET AL., 2016) en que se propone un menor uso de aires acondicionados, una disminución del tráfico urbano, etc. Además, existen estudios que presentan a la vegetación como herramienta para la mitigación de la ICU, KLEEREKOPER ET AL. (2012) mencionan cuatro tipos diferentes de aplicación en las áreas urbanas: bosques urbanos (parques), árboles de alineación, verde en patios privados, techos 
y fachadas verdes. En este sentido, Schмidt (2006) demostró que la vegetación tiene un efecto de enfriamiento promedio de $1 \mathrm{a} 4.7^{\circ} \mathrm{C}$, que se extiende de 100 a $1000 \mathrm{~m}$ en una zona urbana, aunque este alcance depende de la disponibilidad de agua de las plantas y árboles que se van a incorporar. En cuanto a la morfología construida, BouRBIA y AwBI (2004a, 2004b) estudiaron el efecto que ejercen los indicadores H/W (Altura sobre Ancho de Calle), SVF (Factor de Visión de Cielo) y la orientación sobre la temperatura superficial de un conjunto de cañones urbanos en ciudades áridas. Otros abordajes científicos han avanzado parcialmente a partir de investigaciones que demostraron, a escala de barrio, que la forma urbana tiene un fuerte impacto en las temperaturas diurnas (MIDDEL ET AL., 2014; Middel ET AL., 2015). A su vez Sosa ET AL. (2017) consideran la forma de agrupamiento de las edificaciones "trama + perfil urbano" como estrategia de planificación para contribuir al enfriamiento de las temperaturas del aire urbano. Las técnicas de mitigación apuntan a equilibrar el balance de energía de las ciudades, colaboran con las pérdidas térmicas y disminuyen las respectivas ganancias. Por esas razones es preciso un diagnóstico completo de las características del territorio que se va a intervenir y en qué etapa de desarrollo urbano se encuentra el sector para decidir qué estrategias de mitigación son necesarias.

A partir de lo descrito precedentemente, este trabajo tiene como objetivo analizar cómo las distintas formas de urbanizar el área de piedemonte han modificado el microclima del territorio. El objetivo final es evaluar el impacto que tienen las distintas variables de diseño involucradas en los modelos de desarrollo urbano sobre el comportamiento térmico del área de estudio.

\section{Metodología}

Para diagnosticar la condición térmica de los modelos urbanos presentes en el área de piedemonte se realizaron las siguientes etapas: a) caracterización de conjuntos urbanos existentes en el área de piedemonte, b) método de evaluación de los conjuntos urbanos analizados mediante simulaciones dinámicas con el empleo del software ENVI-met; c) ajuste de los resultados del comportamiento térmico entre el modelo simulado y medido para un conjunto urbano existente y d) descripción del modelo físico empleado en la simulación. 


\subsection{Caracterización de conjuntos urbanos existentes en el área de piedemonte}

De lo discutido previamente en el área de piedemonte conviven dos modelos de desarrollo urbano claramente identificables con diferencias de diseño e inserción urbana: urbanizaciones de origen público y urbanizaciones de origen privado. Se presenta para cada uno de ellos una descripción de sus principales características. Para caracterizar cada barrio se consideraron de cada conjunto aspectos morfológicos, indicadores urbanos y edilicios tales como superficie total, cantidad de viviendas, altitud, pendiente, trazado de la trama, ancho de canales viales, dimensiones de manzanas y lotes, factor de ocupación del suelo, retiros y tipo de especies forestales de alineación.

\subsubsection{Urbanizaciones de origen público}

Se presentan las principales características de dos barrios de origen público — barrios Municipal y Estanzuela - representativos del modelo de desarrollo estatal imperante en el área de estudio.

Barrio Municipal: año de creación 1976. Localizado en el departamento de Las Heras, ubicado al noroeste del AMM (Lat. Sur $32^{\circ} 56^{\prime}$ y Long. Oeste $68^{\circ} 52^{\prime}$ ), a una altitud de $800 \mathrm{msnm}$, posee una superficie de $543.158 \mathrm{~m}^{2}$, con un total de aproximado de 1357 viviendas. El trazado de este barrio es del tipo ortogonal, que combina manzanas rectangulares de orientación nor-nordeste-sur-sureste y oeste-suroeste-este-nordeste, implantadas en un terreno con una pendiente natural del $15 \%$. Sus vías de acceso principales —de $30 \mathrm{~m}$ de ancho- son periféricas y tienen una orientación nor-nordeste-sur-sureste. Posee las siguientes características morfológicas: manzanas de $150 \mathrm{~m}$ de largo y $40 \mathrm{~m}$ de ancho, calles de $20 \mathrm{~m}$ las secundarias y $16 \mathrm{~m}$ de ancho las terciarias, lotes de $200 \mathrm{~m}^{2}$ de superficie, Factor de Ocupación de Suelo (FOS) de 0,55, retiros: frontal de $2 \mathrm{~m}$, lateral de 5,50 m solo en uno de sus lados, ya que el diseño del barrio consta de viviendas apareadas. La forestación de calles está conformada por especies del tipo Melia azedarach (paraíso), árbol de segunda magnitud, caducifolio, con una altura máxima de $15 \mathrm{~m}$, dimensiones de copa que varían de 4 a $8 \mathrm{~m}$ de diámetro y una permeabilidad solar media de $48 \%$ en verano (CANTÓN ET AL., 1994). 
Barrio Estanzuela: año de creación 1985. Localizado en el departamento de Godoy Cruz, ubicado al suroeste de AMM, a una altitud de $907 \mathrm{msnm}$. Cubre una superficie aproximada de $700.408 \mathrm{~m}^{2}$, con un total de 1750 viviendas, siendo uno de los barrios públicos más grandes de la provincia, que surgió como respuesta del Estado provincial a las necesidades habitacionales derivadas del terremoto de 1985. El trazado del barrio es predominantemente de tipo ortogonal, con manzanas de proporción rectangular de orientación noroeste-sureste, implantadas en un terreno con una pendiente natural del $15 \%$. El barrio está atravesado por dos vías principales de acceso — de $30 \mathrm{~m}$ de ancho-, de orientación noroeste-sureste y oeste-este. Desde el punto de vista morfológico, el barrio presenta manzanas de $250 \mathrm{~m}$ de largo y $40 \mathrm{~m}$ de ancho, calles de $20 \mathrm{~m}$ de ancho, lotes de $200 \mathrm{~m}^{2}$ de superficie, factor de ocupación del suelo de 0.61 (valor promedio entre la superficie de construcción original y las posteriores ampliaciones) (CASTILLO ET AL., 2015); retiros: si bien el diseño del barrio original previó retiros frontales y laterales, estos fueron ocupados en el tiempo debido al incremento de superficie de las viviendas como resultado del crecimiento poblacional. La forestación de calles está conformada por especies del tipo Schinus molle (aguaribay), árbol de segunda magnitud, perenne, con una altura máxima de $15 \mathrm{~m}$, dimensiones de copa que varían de 4 a $8 \mathrm{~m}$ de diámetro y una permeabilidad solar alta en verano.

Si bien ambos barrios presentan un modelo de desarrollo de tipo racional, se diferencian entre sí respecto de su implantación, la orientación de las manzanas, los retiros, el tipo de arbolado en alineación de calles y la estructura de las vías primarias de circulación. El barrio Municipal está ubicado al norte de la ciudad, combina manzanas de orientación nor-noreste-sur-sureste y oeste-suroeste-este-noreste; presenta retiro frontal y lateral en una de sus caras, arbolado conformado por especies del tipo Melia azedarach —árbol caducifolio de media permeabilidad a la radiación solar-y avenidas principales de carácter periférico. El barrio Estanzuela se localiza al sur de la ciudad, presenta una orientación de manzanas oeste-este, no posee retiros frontales ni laterales, el arbolado está conformado por especies del tipo Schinus molle -árbol perenne de alta permeabilidad a la radiación solar-y avenidas principales de circulación rápida que cruzan en ambos sentidos al barrio. De lo descrito se desprende que el barrio Estanzuela presenta mayor ocupación del suelo y un mayor acceso al recurso solar en verano, derivado de la orientación de las vías de circulación primaria y secundaria, mayor ancho de calles y del tipo de vegetación utilizada en los canales viales. En la figura 1 se presenta la ubicación en el área de piedemonte de los dos conjuntos urbanos de origen público. 


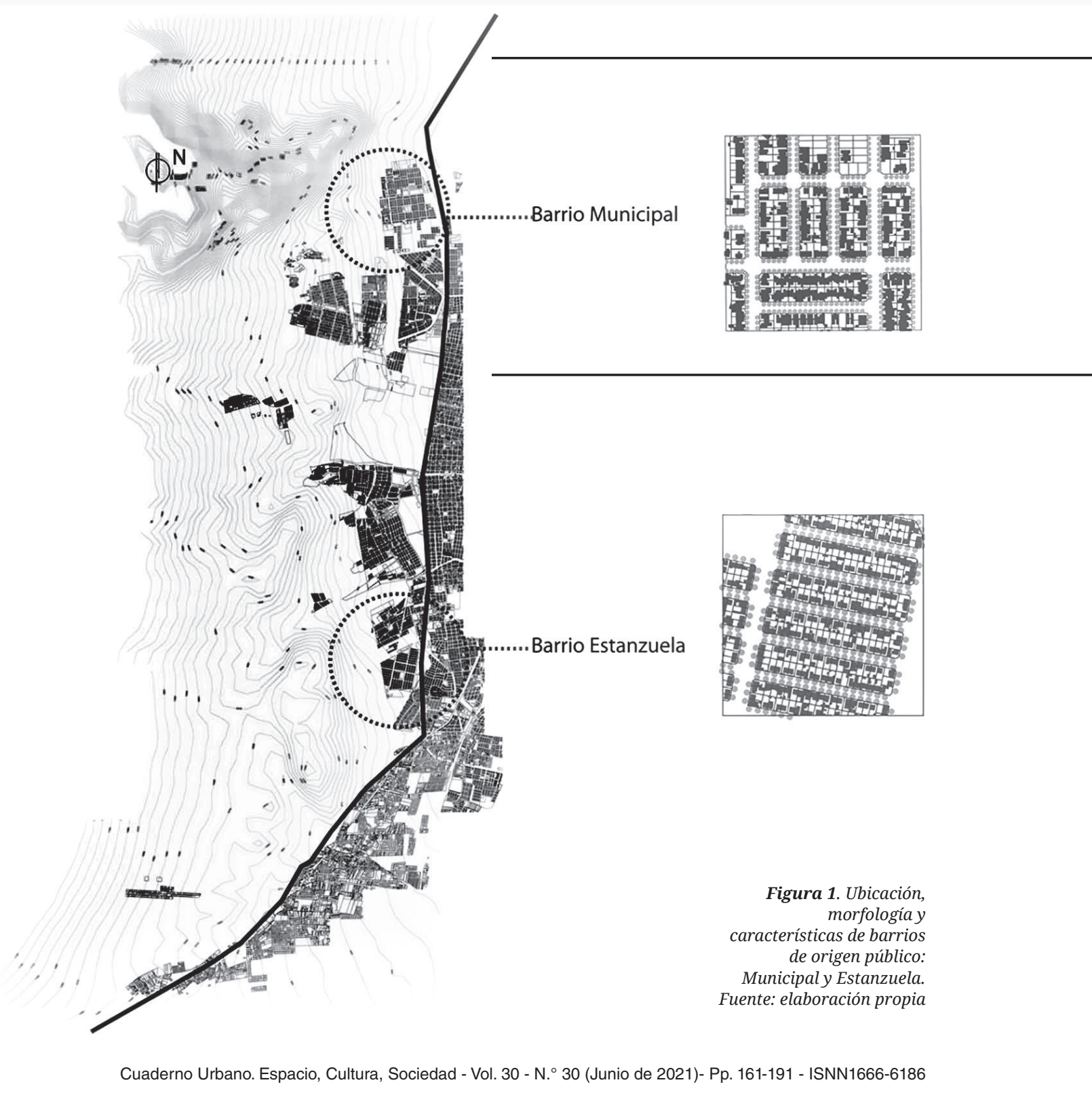




\begin{tabular}{|c|c|c|c|c|c|c|c|c|c|c|}
\hline Barrios & $\underset{\left(\mathrm{m}^{2}\right)}{\text { Superficie }}$ & $\begin{array}{l}\text { Cantidad de } \\
\text { viviendas } \\
\text { (un) }\end{array}$ & $\begin{array}{l}\text { Pen- } \\
\text { diente } \\
(\%)\end{array}$ & $\begin{array}{l}\text { Trazado } \\
\text { urbano y } \\
\text { orientación }\end{array}$ & $\begin{array}{l}\text { Ancho de } \\
\text { canales } \\
\text { viales (m) }\end{array}$ & $\begin{array}{l}\text { Dimensión } \\
\text { manzanas } \\
(\mathrm{m})\end{array}$ & $\begin{array}{l}\text { Dimensión } \\
\text { de lotes }\left(\mathrm{m}^{2}\right)\end{array}$ & FOS & Retiros & Forestación \\
\hline Municipal & 543.158 & 1357 & 15 & $\begin{array}{c}\text { Ortogonal } \\
\text { nor-nordeste } \\
\text { - sur-sureste } \\
\text { y oeste- } \\
\text { suroeste } \\
\text { - este- } \\
\text { nordeste }\end{array}$ & $\begin{array}{c}30 \\
\text { (principales) } \\
-20 \\
\text { (secundarias) } \\
\text { y } 16 \\
\text { (terciarias) }\end{array}$ & $\begin{array}{l}150 \text { largo } \\
40 \text { ancho }\end{array}$ & 200 & 0,55 & $\begin{array}{c}2 \text { frontal } \\
-5,50 \\
\text { lateral }\end{array}$ & $\begin{array}{c}\text { Melia } \\
\text { azedarach: } \\
\text { caducifolio, } \\
\text { permeabilidad } \\
\text { solar media }\end{array}$ \\
\hline Estanzuela & 700.408 & 1750 & 15 & $\begin{array}{l}\text { Ortogonal } \\
\text { noroeste- } \\
\text { sureste y } \\
\text { oeste-este }\end{array}$ & $\begin{array}{c}30 \\
\text { (principales) } \\
-20 \\
\text { (secundarias) }\end{array}$ & $\begin{array}{l}250 \text { largo } \\
40 \text { ancho }\end{array}$ & 200 & 0,61 & - & $\begin{array}{l}\text { Schimus molle: } \\
\text { perenne, } \\
\text { permeabilidad } \\
\text { solar alta }\end{array}$ \\
\hline
\end{tabular}

\subsubsection{Urbanizaciones de origen privado}

Se describen los rasgos principales de dos barrios de desarrollo privado —-barrios Mendoza Norte y Palmares Valley-representativos de uno de los modelos de desarrollo imperantes en el área de estudio.

Barrio Mendoza Norte Country Club, año de creación 2017. Localizado en el departamento de Las Heras, ubicado al noroeste del AMM, a una altitud de $840 \mathrm{msnm}$, posee una superficie de $906.522 \mathrm{~m}^{2}$ y una cantidad aproximada de 500 viviendas. En el trazado del barrio domina la trama racional, si bien en sus bordes presenta tramas del tipo orgánico lineal. El $75 \%$ de las manzanas posee orientación oeste-este, implantadas en un terreno desmontado cuya pendiente natural original era del $30 \%$. De acuerdo con el concepto intrínseco de barrio privado, sus vías de acceso principales son periféricas con orientación norte-sur. Las características urbano-edilicias son las siguientes: manzanas de $200 \mathrm{~m}$ de largo y $70 \mathrm{~m}$ de ancho, calles de $20 \mathrm{~m}$ de ancho, superficie de lotes de $700 \mathrm{~m}^{2}$ (20 m de ancho y $35 \mathrm{~m}$ de profundidad), factor de ocupación del suelo de 0,35 , retiro frontal de $5 \mathrm{~m}$ y retiros laterales de $3 \mathrm{~m}$. La forestación está conformada por especies del tipo Schinus molle (aguaribay) — perenne y con alta permeabilidad solar-.

Barrio Palmares Valley, año de creación 2012. Localizado en el departamento de Luján de Cuyo en la zona de mayor desarrollo urbanístico del AMM, ubicado al suroeste del AMM, a una altitud de $890 \mathrm{msnm}$, posee una superficie de $970.377 \mathrm{~m}^{2}$, con una cantidad de 550 
viviendas. Su trama es de diseño orgánico lineal; el 90 \% de las manzanas tienen orientación nordeste-suroeste. La organicidad de las formas no acompaña el terreno natural; al igual que en el caso del barrio Mendoza Norte Country Club, el terreno ha sido desmontado a partir de una pendiente natural del 25 \%. Así mismo, la vía de acceso principal es de carácter puntual desde un corredor de circulación norte-sur. Las características urbano-edilicias son las siguientes: manzanas de $250 \mathrm{~m}$ de largo y $80 \mathrm{~m}$ de ancho, calles de $20 \mathrm{~m}$ de ancho, superficie de lotes de $800 \mathrm{~m}^{2}$ (20 $\mathrm{m}$ de ancho y $40 \mathrm{~m}$ de profundidad), factor de ocupación del suelo de 0,35, retiro frontal de $2 \mathrm{~m}$ y retiros laterales de $3 \mathrm{~m}$. La forestación está conformada por especies del tipo Acacia visco. Es un árbol caducifolio, con una alta permeabilidad solar puede alcanzar una altura de $12 \mathrm{~m}$ con una copa de diámetro máximo de $12 \mathrm{~m}$.

Ambos barrios presentan diferencias sustanciales con respecto a la localización, el modelo de desarrollo, la orientación de manzanas y el retiro frontal. El barrio Mendoza Norte está ubicado al norte de la ciudad, posee una estructura de trama del tipo racional, manzanas predominantemente orientadas en sentido oeste-este y retiro frontal de $5 \mathrm{~m}$. El barrio Palmares Valley se localiza al sur de la ciudad, posee una estructura de trama del tipo orgánico lineal, manzanas orientadas en el sentido nordeste-suroeste y retiros frontales de $2 \mathrm{~m}$. Además, presentan diferencias menores respecto del tamaño de manzanas, profundidad de lotes y tipo de forestación de canales viales. Si bien la especie forestal utilizada en ambos barrios es diferente, en ambos casos se trata de especies de alta permeabilidad solar en verano. En la figura 2 se presenta la ubicación en el área de piedemonte de los dos conjuntos urbanos de origen privado.

\begin{tabular}{|c|c|c|c|c|c|c|c|c|c|c|}
\hline Barrios & $\underset{\left(\mathrm{m}^{2}\right)}{\text { Superficie }}$ & $\begin{array}{l}\text { Cantidad de } \\
\text { viviendas } \\
\text { (un) }\end{array}$ & $\begin{array}{l}\text { Pen- } \\
\text { diente } \\
(\%)\end{array}$ & $\begin{array}{l}\text { Trazado } \\
\text { urbano y } \\
\text { orientación }\end{array}$ & $\begin{array}{c}\text { Ancho de } \\
\text { canales } \\
\text { viales (m) }\end{array}$ & $\begin{array}{l}\text { Dimensión } \\
\text { manzanas } \\
(\mathrm{m})\end{array}$ & $\begin{array}{l}\text { Dimensión } \\
\text { de lotes }\left(\mathrm{m}^{2}\right)\end{array}$ & FOS & Retiros & Forestación \\
\hline $\begin{array}{l}\text { Mendoza } \\
\text { Norte } \\
\text { Country } \\
\text { CLub }\end{array}$ & 906.522 & 500 & 30 & $\begin{array}{l}\text { Orgánico } \\
\text { lineal }\end{array}$ & $\begin{array}{c}30 \\
\text { (principales } \\
\text { Norte-Sur) } \\
20 \\
\text { (secundarias) }\end{array}$ & $\begin{array}{l}200 \text { largo } \\
70 \text { ancho }\end{array}$ & 700 & 0,35 & $\begin{array}{l}5 \text { frontal } \\
3 \text { lateral }\end{array}$ & $\begin{array}{l}\text { Schimus molle: } \\
\text { perenne, } \\
\text { permeabilidad } \\
\text { solar alta }\end{array}$ \\
\hline $\begin{array}{l}\text { Palmares } \\
\text { Valley }\end{array}$ & 970.377 & 550 & 25 & $\begin{array}{c}\text { Orgánico } \\
\text { lineal }\end{array}$ & $\begin{array}{c}30 \\
\text { (principales) } \\
20 \\
\text { (secundarias) }\end{array}$ & $\begin{array}{l}250 \text { largo } \\
80 \text { ancho }\end{array}$ & 800 & 0,35 & - & $\begin{array}{l}\text { Parasenegalia } \\
\text { visco: } \\
\text { caducifolia, } \\
\text { permeabilidad } \\
\text { solar alta }\end{array}$ \\
\hline
\end{tabular}


Impacto del diseño de modelos urbanos públicos y privados

sobre la respuesta térmica del sector de piedemonte.

El caso del Área Metropolitana de Mendoza, Argentina

Figura 2. Ubicación en el piedemonte $y$ características de los barrios Mendoza Norte y Palmares Valley. Fuente: elaboración propia

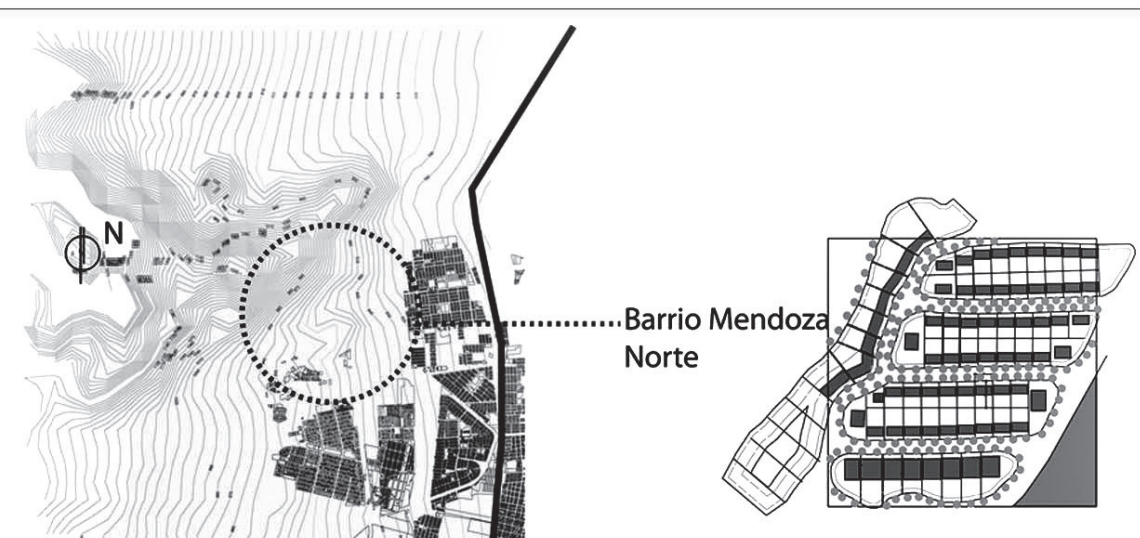


Las diferencias principales entre los barrios de origen público y privado son las dimensiones de los lotes, la profundidad de las manzanas y el factor de ocupación de suelo. En los barrios de origen público, la dimensión de los lotes es de $200 \mathrm{~m}^{2}$, con manzanas de $40 \mathrm{~m}$ de profundidad, mientras que en los lotes de los barrios privados las dimensiones mínimas son de $700 \mathrm{~m}^{2}$ y la profundidad de las manzanas es de $80 \mathrm{~m}$. Otra diferencia sustancial es el factor de ocupación del suelo. Los barrios de origen público han aumentado la ocupación del suelo debido a las ampliaciones que realizaron sus habitantes de 0.31 a 0.61 ; los barrios privados, al tener una normativa interna que restringe las modificaciones de las viviendas, han mantenido el FOS en 0.35

\subsection{Método de evaluación de esquemas urbanos}

Para evaluar el comportamiento de las diferentes formas de urbanizar el área de piedemonte, las acciones se dividieron en etapas: a) monitoreo y selección del caso testigo considerado como representativo del área urbana de piedemonte, b) modelo de simulación (software utilizado), c) ajuste de resultados medidos y simulados y d) descripción del modelo físico empleado en la simulación.

\subsubsection{Monitoreo y selección del caso testigo}

Considerando que los resultados de esta investigación se sustentan en predicciones derivadas de procesos de simulación numérica, es necesario validarlas mediante el ajuste del modelo teórico con el resultado de las mediciones de un caso. Se seleccionó como conjunto residencial referente el barrio Municipal, por contar con un esquema urbano de trama racional, ya que constituye la trama más representativa de todo el piedemonte del AMM (80 \%). El sector donde está inserto este barrio posee una lógica de alto crecimiento y expansión urbana de viviendas de baja densidad, además consiste en uno de los sectores que mayor crecimiento urbano ha sufrido durante la última década Este barrio fue monitoreado mediante una campaña de medición de verano durante el año 2018 (del 2 al 30 de enero). Se adquirieron en forma simultánea los datos de temperatura y humedad relativa del aire cada 15 minutos, mediante sensores del tipo HOBO UX100-003 - Temperature/ Relative Humidity data logger - ubicados en el centro de dos canales viales con distinta orientación: norte-sur y oeste-este, a una altura de $2 \mathrm{~m}$ desde el nivel de la calle (Оке, 2004). Los equipos de medición fueron colocados dentro de una caja perforada de PVC 

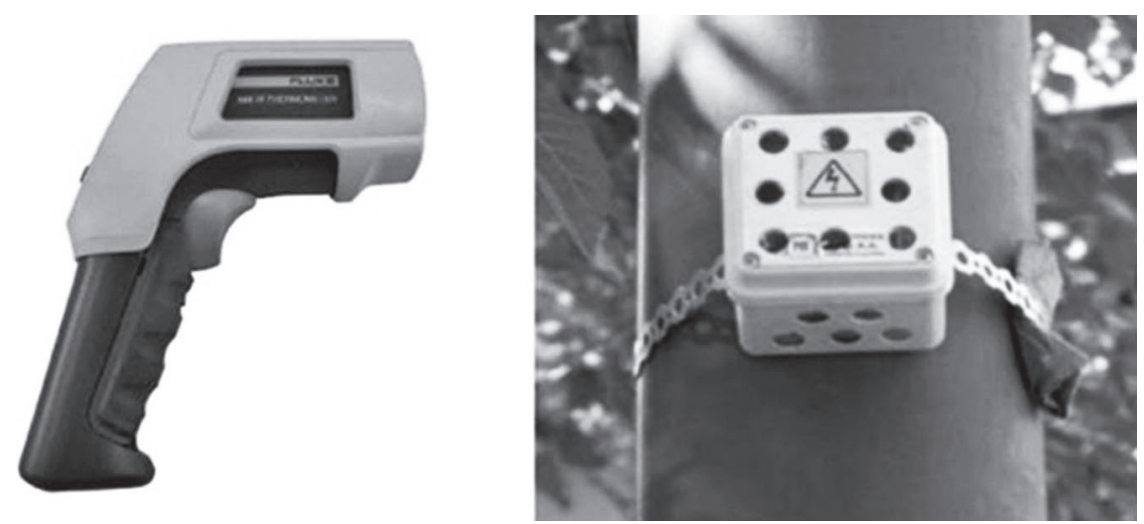

Figura 3. Fotografías del equipamiento utilizado en campañas de medición. Fuente: elaboración propia

blanco, con el objeto de evitar la irradiación y asegurar una adecuada circulación del aire. La figura 3 muestra el equipamiento utilizado para desarrollar las campañas de monitoreo.

\subsubsection{Modelo de simulación}

Los conjuntos urbanos seleccionados se evaluaron micro-climáticamente mediante el uso del software ENVI-met, basado en las leyes fundamentales de la dinámica de fluidos y la termodinámica (BRuse, 1999). Es un modelo computacional 3D que trabaja a escala urbana dentro de un ciclo diario diseñado para simular las interacciones entre el aire y la superficie del entorno urbano con una resolución típica de 0,5 a 10 metros cada 10 segundos. El modelo incluye la simulación de flujos alrededor y entre edificios, procesos de intercambio de calor y vapor de las superficies de suelo y paredes, turbulencia, parámetros de la vegetación, bioclimatología y dispersión de contaminantes. Fue desarrollado por el grupo de trabajo Environmental Modelling Group del Dr. Michael Bruse del Institute of Geography of the University of Mainz, Alemania (http://www.envi-met.com). El uso de ENVI-met está ampliamente validado tanto a nivel internacional como nacional (Tumini, 2012; PeRINI \& MAGLIOCCO, 2014; AlChAPAR, 2014; Stocco, 2016). 
Para la configuración de los modelos teóricos se incorporan los siguientes datos en archivos de entrada principal:

a) Área de entrada: se crea a través de una interfaz gráfica; contiene los datos de los valores del diseño físico del modelo, es decir, la ubicación geográfica del esquema urbano, la forma y dimensiones de la edilicia, la vegetación, distribución de materiales de superficie y tipos de suelo y además la ubicación de los receptores en la grilla planteada.

b) Base de datos: se incorporan en esta sección los datos y características de la forestación y la composición del suelo. La especie forestal utilizada para el ajuste del caso testigo fue la denominada "TH" de la librería PLANTS.DAT de ENVI-met; la altura se determinó en 15 $\mathrm{m}$ por corresponder con el desarrollo promedio de un forestal de $2^{\circ}$ magnitud. Las características de esta tipología forestal son las siguientes: 400 de resistencia mínima estomática, 0.20 de albedo de onda corta de la hoja de la planta, $2 \mathrm{~m}$ de profundidad total de la zona radicular, LAD (Leaf Area Density) de 0.40 a 1.80 de densidad del área foliar en $\mathrm{m}^{2} / \mathrm{m}^{3}$ y RAD (Root Area Density) de 0.10 de densidad del área de la raíz en $\mathrm{m}^{2} / \mathrm{m}^{3}$. Para la simulación de los conjuntos urbanos con forestación de alta permeabilidad solar, se utilizó la especie forestal denominada "DO", a la que le corresponde un valor de LAD de 2.0 a 0.25 de densidad del área foliar en $\mathrm{m}^{2} / \mathrm{m}^{3}$, siendo los valores del resto de las variables las presentadas previamente. La composición del suelo se configuró en la librería (SOILS.DAT y PROFILS. DAT) ingresando la caracterización por capa en profundidad del suelo con el objetivo de representar el perfil de suelo del área de piedemonte de Mendoza.

c) Definición del archivo de configuración: incluye las variables meteorológicas y condiciones del entorno del territorio en el que se evalúan los casos de estudio (velocidad y dirección de viento, rugosidad, temperatura atmosférica inicial, humedad específica y humedad relativa).

\subsection{Ajuste de resultados medidos y simulados}

Con el fin de validar y dar confianza estadística a los resultados de las simulaciones de los conjuntos urbanos, se realizó un ajuste del conjunto urbano monitoreado (barrio Municipal) con el modelo numérico. Para ello se escogió del período monitoreado en el verano de 2018 el día 15 de enero, dado que presentó condiciones meteorológicas representativas de un día típico en el área de estudio, es decir, altas temperaturas y heliofanía, baja humedad relativa y velocidad de viento. El período de estabilización encontrado es de 72 horas; este período es congruente con los obtenidos por WANG y AKBARI (2016). 


\section{Impacto del diseño de modelos urbanos públicos y privados \\ sobre la respuesta térmica del sector de piedemonte.}

El caso del Área Metropolitana de Mendoza, Argentina

En la tabla 2 se presentan los datos de entrada del archivo "Configuration File", para el ajuste del esquema de trama racional. Esta se divide en tres categorías de datos: meteorológicos, edificación y suelo. Los datos meteorológicos y de edificación de entrada se mantienen constantes en los ajustes, ya que son condiciones climáticas a nivel meso-escala, y la tecnología y materialidad de las viviendas es la misma. Por último, se ajustaron el perfil de composición de suelo y las temperaturas superficiales de este, de las capas inicial y media.

Tabla 2

\begin{tabular}{lc}
\hline Valores archivo configuración ENVI-met & \\
\hline Parámetros para configuración Envi-met & valores \\
\hline Meteorológicos & * valores por defecto de ENVI-met \\
\hline Velocidad viento a $10 \mathrm{~m}$ sobre suelo $(\mathrm{m} / \mathrm{s})$ & 3.5 \\
\hline Dirección viento $\left(0^{\circ}: \mathrm{N}, 90^{\circ}:\right.$ E, , $\left.180^{\circ}: \mathrm{S}, 270^{\circ}: \mathrm{W}\right)$ & $135^{\circ}$ \\
\hline Rugosidad z0 & 0.1 \\
\hline Factor de ajuste solar & 1.5 \\
\hline Temperatura atmosférica inicial $(\mathrm{K})$ & 298 \\
\hline Humedad específica a $2500 \mathrm{msnm}(\mathrm{g} / \mathrm{kg}-1)$ & 2.8 \\
\hline Humedad relativa a $2 \mathrm{~m}(\%)$ & 37 \\
\hline Edificación & 297 \\
\hline Temperatura interior $(\mathrm{K})$ & 2 \\
\hline Conductividad pared $\left(\mathrm{W} / \mathrm{m}^{2} \mathrm{~K}\right)$ & 0.78 \\
\hline Conductividad techo $\left(\mathrm{W} / \mathrm{m}^{2} \mathrm{~K}\right)$ & 0.3 \\
\hline Albedo pared & 0.2 \\
\hline Albedo techo & $293 \mathrm{~K}^{*}$ \\
\hline Suelo & $290 \mathrm{~K}^{*}$ \\
\hline Temperatura superficial, capa inicial $(0-20 \mathrm{~cm})$ & $290 \mathrm{~K}^{*}$ \\
\hline Temperatura superficial, capa media $(20-50 \mathrm{~cm})$ & $20 \%^{*}$ \\
\hline Temperatura superficial, capa profunda $(<50 \mathrm{~cm})$ & $35 \%^{*}$ \\
\hline Humedad relativa, capa inicial $(0-20 \mathrm{~cm})$ & $60 \%^{*}$ \\
\hline Humedad relativa, capa media $(20-50 \mathrm{~cm})$ & \\
\hline Humedad relativa, capa profunda $(<50 \mathrm{~cm})$ & \\
\hline
\end{tabular}

Fuente: elaboración propia 
En la tabla 3 se presentan las curvas de ajuste del caso simulado con los valores de temperatura del aire máxima, mínima, promedio y amplitud, como así también se muestran los valores de SVF (Sky View Factor) y los seis indicadores estadísticos utilizados para estimar el grado de precisión del modelo numérico simulado versus el monitoreado. Esto permite demostrar la confiabilidad de los resultados arrojados por la simulación. La identificación y cuantificación de los errores se realizó mediante la determinación del coeficiente de correlación ajustado o determinación (R2), el error de polarización media (MBE), el error absoluto medio (MAE), el error medio porcentual absoluto (MAPE), el error cuadrático medio (RSME), el error cuadrático medio sistemático (RSMEs) y el error cuadrático medio aleatorio (RSMEu). Cada indicador expresa el grado de exactitud o error del modelo bajo diversas perspectivas; MAE y RMSE indican la magnitud del error promedio, pero no proporcionan información sobre el tamaño relativo de la diferencia media entre los valores observados y los valores predichos. MBE describe la dirección del sesgo de error; su valor está

Tabla 3

\section{Curva de ajuste del caso simulado y los indicadores estadísticos}

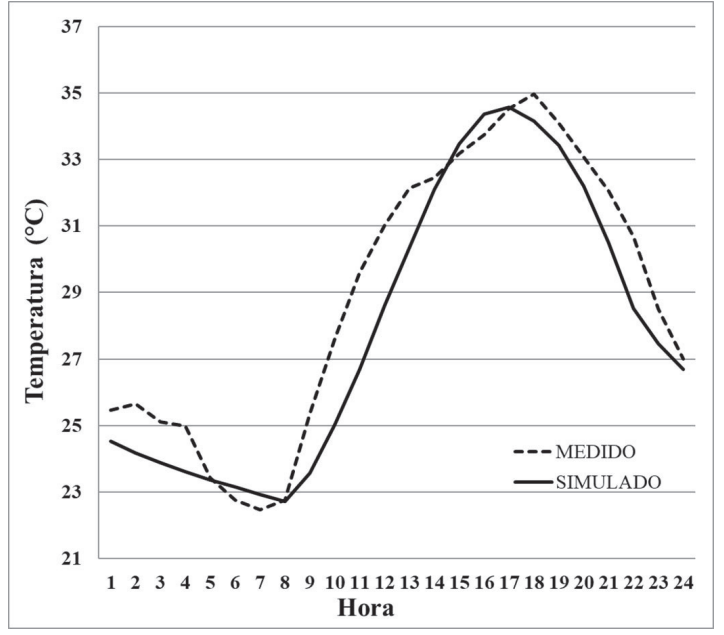

\begin{tabular}{|c|c|c|c|c|c|}
\hline $\mathrm{T}^{\mathrm{o}}\left({ }^{\circ} \mathrm{C}\right)$ & Máxima & Mínima & Prom. & Ampl. & SVF \\
\hline Simulado & 34.56 & 22.72 & 27.52 & 11.84 & 0.69 \\
\hline Medido & 34.96 & 22.48 & 28.86 & 12.48 & 0.62 \\
\hline Delta & 0.39 & -0.24 & -1.34 & 0.64 & 0.07 \\
\hline
\end{tabular}

Fuente: elaboración propia 
Impacto del diseño de modelos urbanos públicos y privados sobre la respuesta térmica del sector de piedemonte.

El caso del Área Metropolitana de Mendoza, Argentina

Tabla 4

Ubicación de receptores en las tramas urbanas

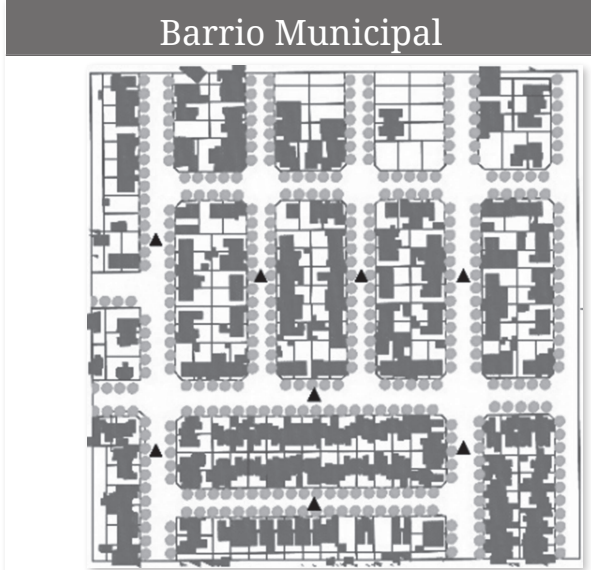

Barrio Estanzuela

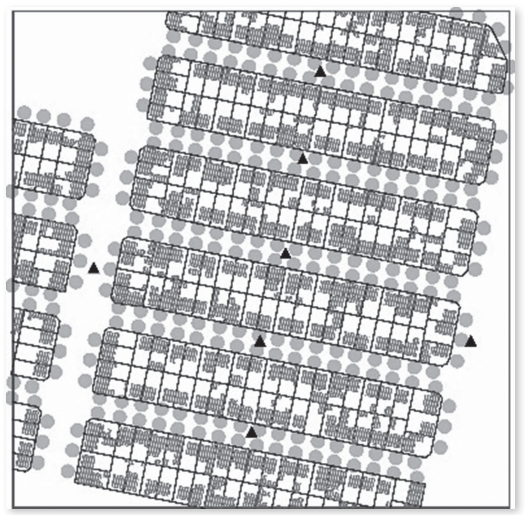

Barrio Mendoza Norte

Barrio Palmares Valley
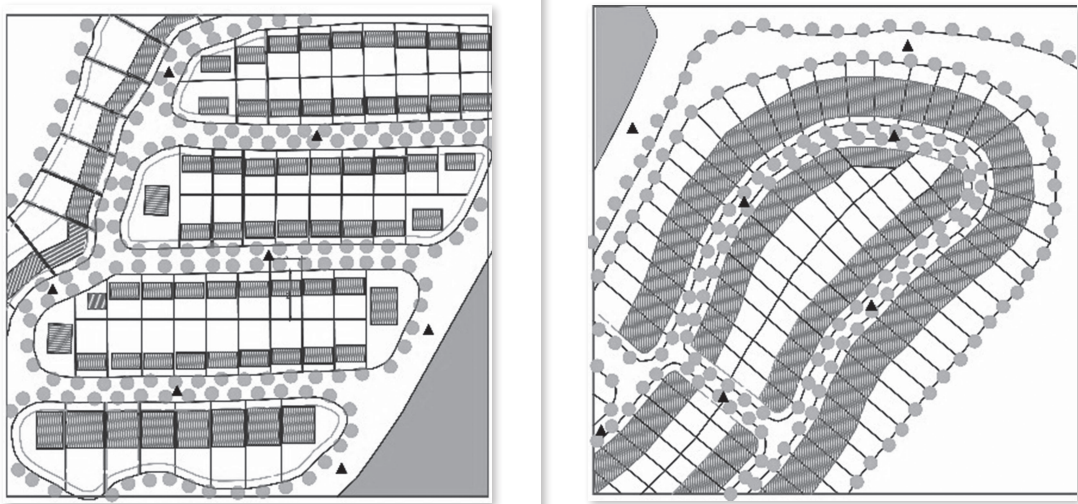

Ubicación receptores en modelo teórico

Fuente: elaboración propia 
relacionado con la magnitud de los valores utilizados; un MBE negativo se produce cuando las predicciones son más pequeñas en valor que las observaciones. MAPE expresa al error como un porcentaje, suponiendo que es 5 , el pronóstico está errado en un $5 \%$. Como este número es un porcentaje, puede ser más fácil de entender que los otros estadísticos.

\subsection{Descripción del modelo físico empleado en la simulación}

Sobre el modelo ajustado y validado se realizaron simulaciones de los conjuntos urbanos públicos y privados. Para la obtención de datos que evidencian el efecto de la trama sobre la temperatura del aire, se ubicaron siete receptores en los canales viales urbanos de cada conjunto. En la tabla 4 se puede observar la ubicación de cada sensor con respecto al canal vial. Las simulaciones se realizaron con una superficie de referencia de 300 x $300 \mathrm{~m}$; la grilla es de 100 x 100 x 30; por lo tanto, la resolución del área es de 3 x 3 x 3 m y el número de grillas totales es de $\mathrm{x}: 86 ; \mathrm{y}: 86 ; \mathrm{z}: 30$.

\section{Resultados}

Se realizó un análisis comparativo de temperaturas del aire promedio de máximas, mínimas y medias de todos los sensores presentes en cada uno de los modelos físicos evaluados para un día típico de verano (15 de enero), y así identificar el esquema urbano que brinda la mejor respuesta térmica; además se realizó un análisis de gráficos del tipo box-plot para identificar la variabilidad de la respuesta térmica de los barrios analizados.

\subsection{Análisis comparativo de temperaturas del aire promedio máximas, mínimas y medias}

A continuación, se presentan los resultados de las simulaciones realizadas con el software ENVI-met. Se exponen los valores de las temperaturas promedio de todos los receptores que corresponden a la condición de máxima, mínima y media de la curva de temperatura del aire. Las temperaturas promedio fueron calculadas a partir de los resultados arrojados por los receptores colocados en las cuatro tramas simuladas — dos correspondientes a los barrios de origen público y dos a los barrios de origen privado—. A partir de la evaluación térmica de cada barrio, se realizaron análisis comparativos entre ellos: a) barrios de origen público, b) barrios de origen privado y c) análisis comparativo entre los cuatro barrios analizados. 
Impacto del diseño de modelos urbanos públicos y privados

sobre la respuesta térmica del sector de piedemonte.

El caso del Área Metropolitana de Mendoza, Argentina

Tabla 5

Temperaturas promedio de las propuestas analizadas

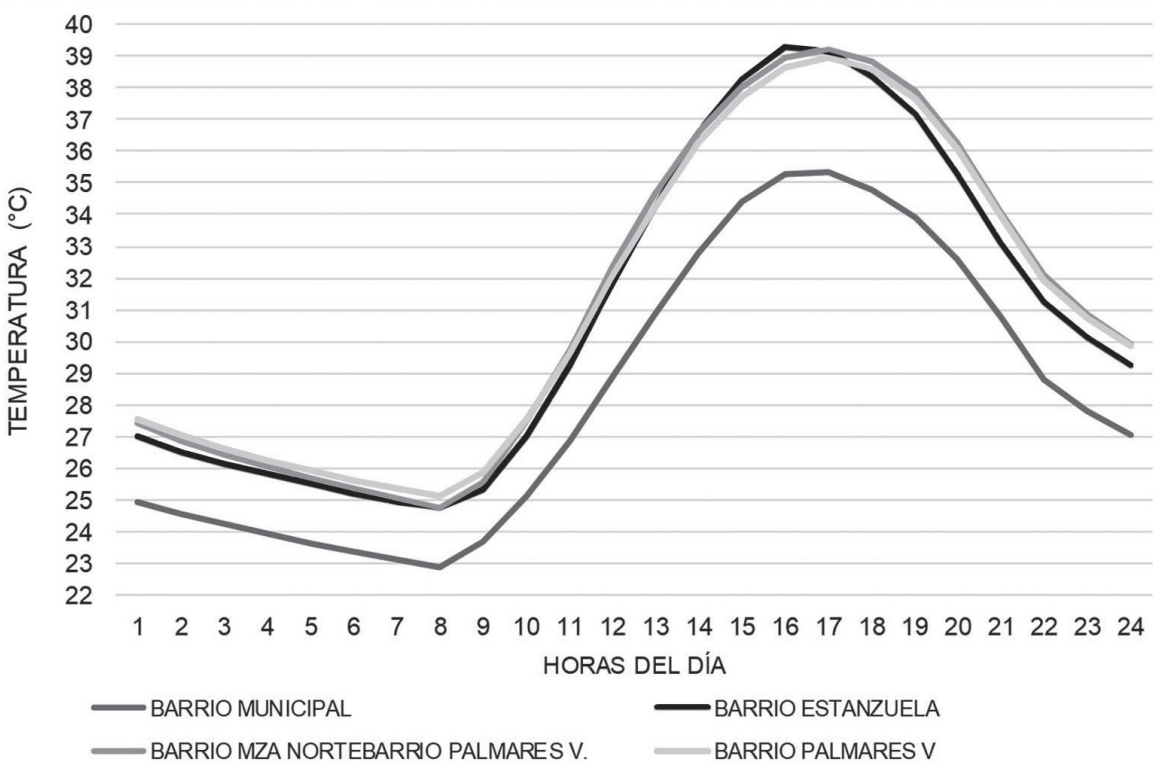

\begin{tabular}{|c|c|c|c|c|}
\hline $\begin{array}{l}\text { Conjuntos } \\
\text { urbanos }\end{array}$ & 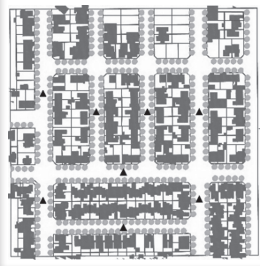 & \begin{tabular}{|c|c|} 
& \\
3 &
\end{tabular} & 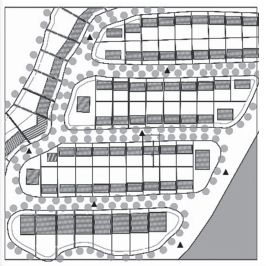 & \\
\hline & B.o Municipal & B.o Estanzuela & B.o Mza. Norte & B.o Palmares V \\
\hline Máxima & 35,36 & 39,24 & 39,20 & 38,95 \\
\hline Mínima & 22,89 & 24,72 & 24,75 & 25,15 \\
\hline Promedio & 28,38 & 30,95 & 31,31 & 31,28 \\
\hline
\end{tabular}

Fuente: elaboración propia 


\subsubsection{Barrios de origen público}

En el análisis comparativo del comportamiento de los barrios de origen público -barrio Municipal vs. barrio Estanzuela - (tabla 5), se puede observar que el barrio Municipal es el que mejor desempeño térmico posee en todas las temperaturas, alcanzando una diferencia con el barrio Estanzuela de hasta $4^{\circ} \mathrm{C}$ en la temperatura máxima, de $2^{\circ} \mathrm{C}$ en la temperatura mínima y $2.5^{\circ} \mathrm{C}$ en la media. Estas diferencias térmicas son el resultado del balance térmico día-noche. Durante el día la ganancia solar está relacionada con el efecto combinado de tres variables: orientación, ancho del canal vial y permeabilidad del arbolado de alineación de calles. En el caso del barrio Municipal, el 75 \% de los canales viales tienen orientación nor-nordeste-sur-sureste y oeste-suroeste-este-nordeste y ancho preponderante de $16 \mathrm{~m}$, forestados por una especie de permeabilidad media. Esta conjunción de variables reduce la exposición solar del conjunto respecto del barrio Estanzuela, en el cual, si bien se presenta una adecuada orientación de sus canales viales, el ancho de estos es mayor $(20 \mathrm{~m})$, al igual que la permeabilidad de los forestales. La mayor ganancia solar asociada a una característica de suelo con alta inercia térmica representativa del área de piedemonte genera como resultado un aumento en la temperatura del aire. A pesar de que el barrio Estanzuela por sus características presenta mejores condiciones para el enfriamiento radiativo nocturno, estas no son suficientes para compensar el impacto del acceso al sol durante el día. De lo descrito se desprende que en urbanizaciones de piedemonte es más importante controlar el impacto de la radiación solar durante las horas del día, debido a que sus posibilidades de enfriamiento son limitadas por la mayor inercia térmica de la composición de suelo; esto implica tender a la compacidad de sus formas.

\subsubsection{Barrios de origen privado}

De la comparación de las curvas de temperatura del aire entre los barrios privados Mendoza Norte y Palmares Valley, se pueden observar temperaturas máximas semejantes y mínimas con diferencias del orden de $0.50^{\circ} \mathrm{C}$, siendo el barrio Mendoza Norte levemente más fresco durante la noche. Si bien ambas urbanizaciones presentan igual ancho de calle $(20 \mathrm{~m})$ y tipo de forestación (alta permeabilidad solar), sus diferencias en el tipo de trama (racional y orgánica) y su orientación (oeste-este y noreste-suroeste) no impactan en el comportamiento térmico debido al bajo factor de ocupación de suelo (0.35) y sus retiros frontales (5 m y $2 \mathrm{~m}$ ). En consecuencia, estos factores que potencian el acceso al sol —variable responsable de las temperaturas del aire—-son determinantes del comportamiento térmico. 


\subsubsection{Comparación de barrios de origen público vs. privado}

Teniendo en cuenta el contraste de la respuesta térmica de los conjuntos urbanos de origen público y privado, se observa que los barrios de origen público presentan mayor eficiencia térmica asociada a la compacidad de las formas. Esta característica es más frecuente en estos barrios dado que el diseño de los barrios privados está orientado hacia un esquema del tipo abierto guiado por el concepto de "barrio jardín". El principio de diseño de barrios privados ofrece posibilidades desde el punto de vista de una mayor disponibilidad de suelo para asegurar una adecuada superficie de infiltración, lo que permite mitigar el riesgo aluvional característico del sector de piedemonte y controlar el impacto de la urbanización a partir de disponer de espacios abiertos para implementar estrategias de enverdecimiento. Sin embargo, es evidente que el diseño de las áreas verdes requiere ser reformulado en función de un enfoque bioclimático que permita un mayor control del acceso al sol, dado que en suelos de alta inercia térmica, como es el caso del piedemonte, la ganancia solar comanda la temperatura del aire del espacio urbano. Además, es importante considerar que el mejor comportamiento térmico de los barrios públicos resultante de sus variables de diseño urbano-edilicias debe ser equilibrado con otros factores que impone el contexto de piedemonte, entre ellos un menor sellamiento de superficies para garantizar la infiltración de suelos.

\subsection{Análisis de la distribución de las temperaturas}

Para contrastar el comportamiento de los diferentes sectores de los conjuntos urbanos analizados — representados por los distintos receptores en el modelo de simulación-, se realizaron gráficos del tipo box-plot, que muestran la variabilidad del comportamiento microclimático.

\subsubsection{Temperatura máxima}

En la figura 4 se observa que la mayor variabilidad de las temperaturas máximas está asociada a urbanizaciones cuya estructura responde a un esquema del tipo racional que alcanza diferencias superiores a los $2^{\circ} \mathrm{C}$ en las estructuras que presentan mayor compacidad (B. Municipal $\mathrm{Q} 1=34.0^{\circ} \mathrm{C}-\mathrm{Q} 3=36.20^{\circ} \mathrm{C}$; B. Estanzuela $\mathrm{Q} 1=36.84^{\circ} \mathrm{C}-\mathrm{Q} 3=38.90^{\circ} \mathrm{C}$ ) y de $1^{\circ} \mathrm{C}$ en las más abiertas (B. Mendoza Norte $\left.\mathrm{Q} 1=37.96^{\circ} \mathrm{C}-\mathrm{Q} 3=39.16\right)$. Finalmente, la menor variabilidad $\left(0.6^{\circ} \mathrm{C}\right)$ se presenta en el B. Palmares Valley $\left(\mathrm{Q} 1=38.27^{\circ} \mathrm{C}-\mathrm{Q} 3=38.90^{\circ} \mathrm{C}\right.$ ), cuya estructura es de tipo orgánica. 


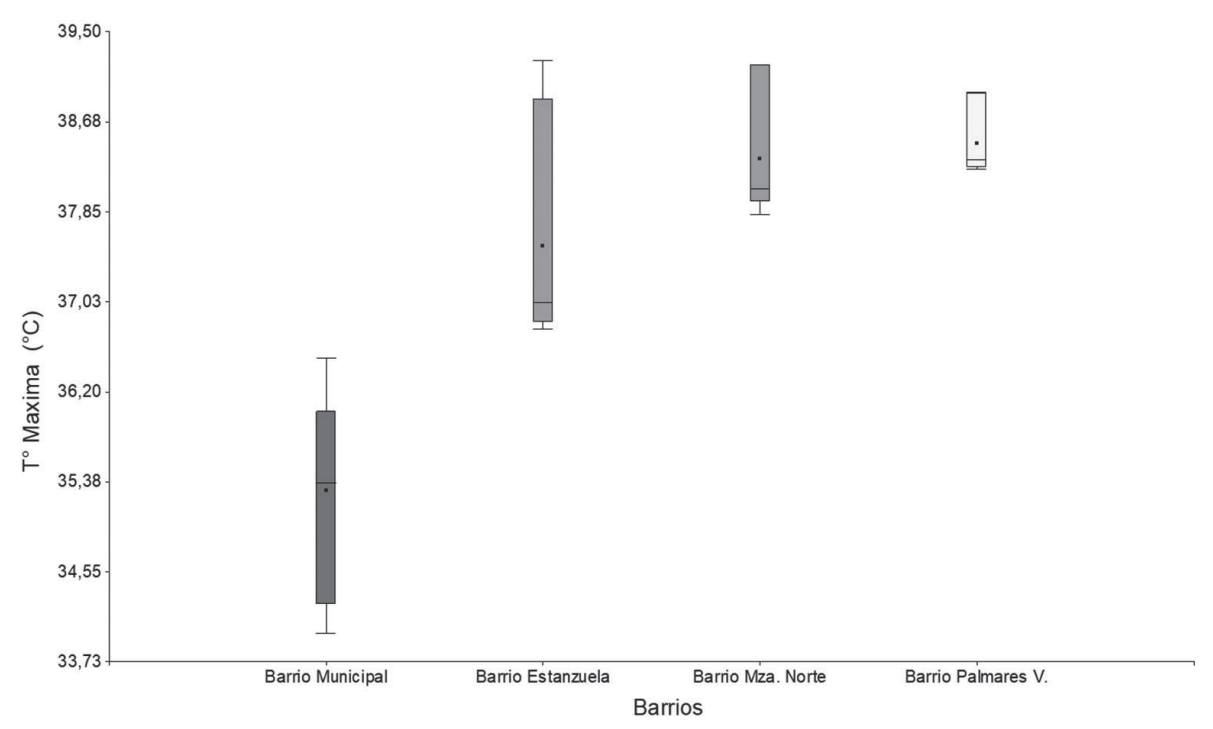

Figura 4. Gráfico box-plot para temperaturas máximas. Fuente: elaboración propia

\subsubsection{Temperatura mínima}

$\mathrm{Al}$ analizar el diagrama de variabilidad de las temperaturas mínimas (figura 5), se puede observar que es del orden de $0.5^{\circ} \mathrm{C}$ para el caso del B. Municipal (Q1 $=22.55^{\circ} \mathrm{C}-\mathrm{Q} 3=23.10$ ${ }^{\circ} \mathrm{C}$ ) y de menor impacto para el resto de los barrios analizados (B. Estanzuela Q1 $=24.52^{\circ} \mathrm{C}$ - Q3 $=24.72^{\circ} \mathrm{C}$; B. Mendoza Norte $\mathrm{Q} 1=24.76^{\circ} \mathrm{C}-\mathrm{Q} 3=24.80^{\circ} \mathrm{C}$ y B. Palmares $\mathrm{Q} 1=25.09^{\circ} \mathrm{C}-$ $\mathrm{Q} 3=25.11^{\circ} \mathrm{C}$ ). La baja variabilidad de las temperaturas mínimas refleja que la homogeneidad de formas hacia el interior de los complejos urbanos analizados (como consecuencia de sus variables de diseño: tipo de trama, orientación, ancho de canal vial, tipo de forestación, etc.) no impacta de forma significativa sobre la distribución de las temperaturas mínimas de los distintos sectores que componen el conjunto. 


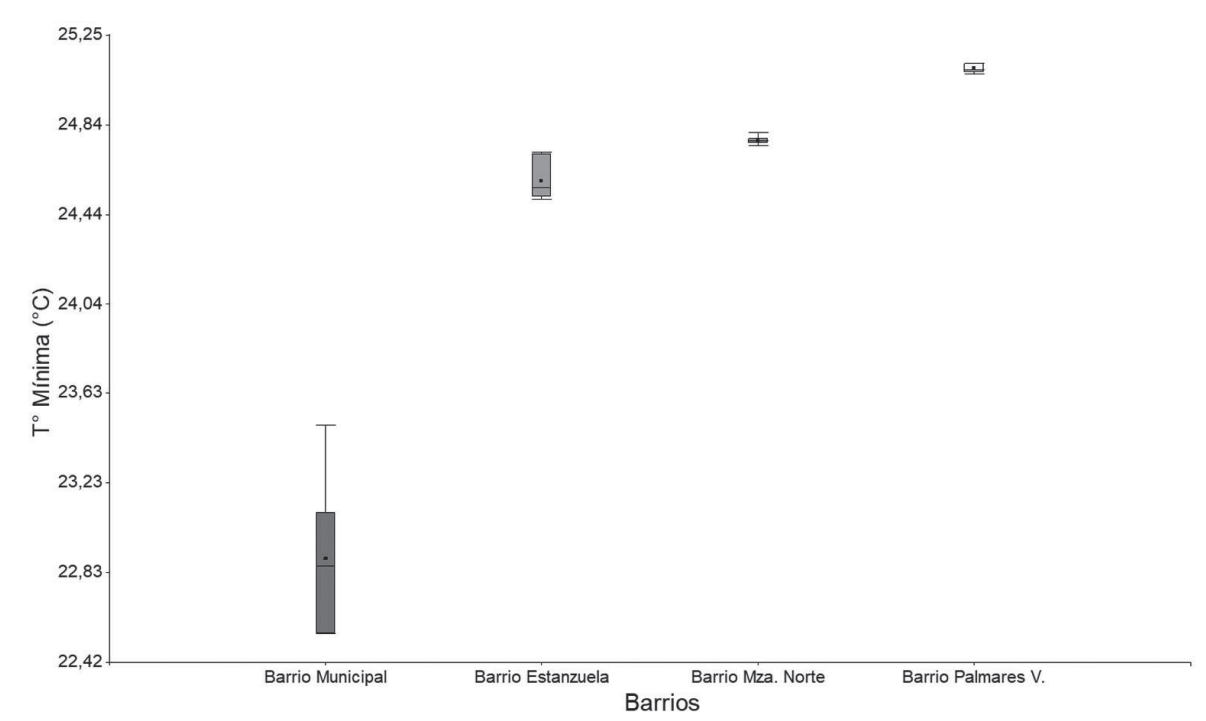

Figura 5. Gráfico box-plot para temperaturas mínimas. Fuente: elaboración propia

\section{Conclusión}

El desarrollo urbano público y privado sobre el área de piedemonte de Mendoza ha originado situaciones de fragmentación del territorio y ha superpuesto funciones que no siempre son compatibles entre sí. Si bien se elaboró un conjunto de leyes y planes de ordenamiento territorial que intentan ordenar el crecimiento urbano en este sector, los emprendimientos urbanísticos no responden a los criterios y pautas que la normativa presenta. Esta situación se destaca en el diseño urbano de barrios de origen público - posteriores a la sanción de las respectivas leyes - que se han implantado en esta área de alta fragilidad, y cuyo diseño responde a las características de llanura. Es por ello que para su construcción se realizaron grandes movimientos de tierra que desmontaron las laderas de las montañas adyacentes a 
la ciudad y modificaron completamente su condición natural. Esta situación ha generado una serie de impactos ecológicos y empobrecido el micro-clima del sector.

Ante este contexto, en el presente trabajo se realizó un análisis comparativo de esquemas urbanos existentes en áreas de piedemonte para evaluar la relación que existe entre el diseño de diferentes conjuntos urbanos y cómo este impacta en el comportamiento micro-climático del área de piedemonte. Los resultados obtenidos del análisis comparativo de los distintos esquemas urbanos muestran que la estrategia de mayor impacto en el comportamiento térmico de una urbanización emplazada en un contexto de piedemonte es el control de la radiación solar. En este sentido, variables de diseño como el ancho de los canales viales y el porcentaje de permeabilidad de los forestales, asociados a la tipología y orientación de la trama, mostraron un impacto significativo en la respuesta térmica del conjunto.

En el caso de los barrios públicos, la estrategia de control solar está asociada a la compacidad de las formas. Sin embargo, esta estrategia debe compatibilizarse con otras condicionantes derivadas de las características propias del piedemonte que demandan principalmente preservar la topografía del terreno y controlar el sellamiento del suelo. En los barrios privados el concepto del barrio jardín dominante en su diseño deriva en una menor ocupación de suelo. Esta condición demanda regular el acceso al sol mediante la aplicación de otro tipo de estrategias vinculadas con el diseño eficiente de los espacios verdes. Se propone reorientar su diseño de "green inglés" - grandes extensiones de verde que requieren un alto nivel de riego- hacia el concepto de bosque, estructura verde que muestra mayor eficiencia en zonas áridas desde el punto de vista de su capacidad de proporcionar sombra sobre el espacio abierto (Sтоссо ET AL., 2018). De esta manera, se plantea como posible compatibilizar un esquema de urbanización de carácter abierto que dé una respuesta adecuada desde el punto de vista bioclimático a las características particulares de su área de implantación.

Respecto de la capacidad de enfriamiento de los conjuntos analizados, el comportamiento de las temperaturas mínimas demuestra que el enfriamiento está condicionado por la alta inercia térmica del tipo de suelo presente en el piedemonte. Las temperaturas mínimas se ordenan de acuerdo con los conceptos de diseño que propician las menores temperaturas durante las horas de sol. En investigaciones futuras se prevé analizar el impacto de trabajar sobre la materialidad y tecnología de las urbanizaciones en relación con la forma, como estrategia para potenciar la capacidad de enfriamiento de los conjuntos urbanos del piedemonte. 
Finalmente, de los conjuntos urbanos analizados, el barrio Municipal muestra el mejor comportamiento micro-climático. Dicho comportamiento se logra como consecuencia de un esquema compacto resultante de la orientación predominante de la trama norte-sur que se ha mostrado como superador en investigaciones previas de la UID (SosA ET AL., 2020). Otros indicadores que resultaron beneficiosos son el ancho de canales viales preponderantes de $16 \mathrm{~m}$; el arbolado de alineación con especies de permeabilidad media (restringe la radiación solar durante las horas del día y permite un refrescamiento nocturno) y un factor de ocupación del suelo del orden del 0.55. Estas características resultan en una menor exposición solar del conjunto, lo que impacta en la atenuación de las temperaturas máximas en el orden de los $4^{\circ} \mathrm{C}$ y de las mínimas en el orden de $\operatorname{los} 2^{\circ} \mathrm{C}$. Aunque el barrio Municipal muestra un mejor comportamiento térmico, se debe señalar que para la construcción de este barrio se realizaron desmontes que modificaron las condiciones del terreno natural. Además, presenta un alto sellamiento del suelo que impide la adecuada infiltración de lluvias estivales con sus consecuentes impactos sobre el riesgo aluvional y el desplazamiento de suelos.

En este trabajo se demuestra la importancia del diseño y su impacto en la respuesta térmica del territorio como consecuencia de la combinación de variables urbano-edilicias y esquemas de enverdecimiento de los barrios emplazados en áreas de piedemonte. En este sentido, el ordenamiento territorial requiere tomar en cuenta el enfoque del diseño bioclimático asociado a las particularidades de las áreas de piedemonte en la regulación de su crecimiento, a fin de garantizar el desarrollo sustentable en áreas de alta fragilidad ambiental.

\section{Referencias}

ABRAHAM, M. E., ROIG, F. A. \& SALOMóN, M. (2005). Planificación y gestión del piedemonte al oeste de la Ciudad de Mendoza. Un asunto pendiente. En Conflictos socio-ambientales y políticas públicas en la provincia de Mendoza. OIKOS-Red Ambiental, pp. 267-295.

AGENCIA PROVINCIAL DE ORDENAMIENTO TERRITORIAL (2009). Ley 8051/09 Ley de Ordenamiento Territorial y Usos del suelo. En Provincia de Mendoza, Argentina. http://www.cafedelasciudades.com.ar

ALCHAPAR, N. (2014). Materiales de la envolvente urbana. Valoración de su aptitud para mitigar la isla de calor en ciudades de zonas áridas. Universidad Nacional de Salta. 
BAZANT, J. (2010). Expansión urbana incontrolada y paradigmas de la planeación urbana. Espacio abierto 19 (3), 475-503. https://produccioncientificaluz.org/index. php/espacio/article/view/1405

BOURBIA, F. \& AWBI, H. B. (2004a). Building cluster and shading in urban canyon for hot dry climate: Part 1: Air and surface temperature measurements. Renewable Energy 29-2, 249-262. doi.org/10.1016/S0960-1481(03)00170-8

BOURBIA, F. \& AWBI, H. B. (2004b). Building cluster and shading in urban canyon for hot dry climate: Part 2: Shading simulations. Renewable Energy, 29-2, 291-301. doi.org/10.1016/S0960-1481(03)00171-X

BRUSE, M. (1999). The influences of local environmental design on microclimate. University of Bochum.

CANTÓN, M. A.; CORTEGOSO, J. L. \& DE ROSA, C. (1994). Solar permeability of urban trees in cities of western Argentina. Energy and Buildings, Berkeley, USA. 20 (3): 219-230. doi.org/10.1016/0378-7788(94)90025-6

CASTILLO, A. L.; CORREA, E. N. \& CANTÓN, M. A. (2017). Regulación urbanística de piedemonte en ciudades latinoamericanas. Análisis crítico de la normativa internacional y el marco regulatorio de la Ciudad de Mendoza, Argentina. ACE: Architecture, City and Environment = Arquitectura, Ciudad y Entorno. 12 (35): 39-70. doi:10.5821/ace.12.35.4761.

CASTILLO, A. L.; CORREA CANTALOUBE, E. N. \& CANTÓN, M. A. (2015). Desarrollo urbano en el piedemonte: Comparación crítica de los esquemas urbanos actuales con la regulación vigente en Mendoza, Argentina. Asociación Argentina de Energías Renovables y Ambiente; Averma; 19; 1; 79-92.

CHRYSOULAKIS, N. \& GRIMMOND, C. S. B. (2016). Understanding and reducing the anthropogenic heat emission. In: Santamouris, M. and Kolokotsa, D. (eds.) Urban climate mitigation techniques. Routledge, 27-40.

CORREA, E. (2006). Isla de Calor Urbana. El caso del aérea metropolitana de Mendoza. Universidad Nacional de Salta.

FLORES, E. (2019). Nuevas tecnologías verdes en zonas áridas. Diseño y Evaluación energético-ambiental de sistemas de vegetación de aplicación en envolventes edilicias. Universidad Nacional de Salta.

GEORGESCU, M.; MOREFIELD, P.; BIERWAGEN, B. \& WEAVERET, C. (2014). Urban adaptation can roll back warming of emerging megapolitan regions. National Academy of Sciences. Vol. 111, N. ${ }^{\circ}$ 8, pp. 2909-2914. https://doi.org/10.1073/pnas.1322280111 
GIGLIO, M. L.; RODRÍGUEZ TARDUCCI, R.; CORTIZO, D. \& FREDIANI, J. (2019). Lógicas de ocupación y acceso al suelo en procesos de desarrollo residencial expansivo. El caso de la periferia del partido de La Plata (2003-2016). En Cuaderno Urbano. Espacio, Cultura, Sociedad - Vol. 27 - N. ${ }^{\circ} 27$ - Pp. 053-086. DOI: http://dx.doi.org/10.30972/crn.27274119

GILL, S. E.; HANDLEY, J. F.; ENNOS, A. R. \& PAULEIT, S. (2007). Adapting cities for climate change: the role of the green infrastructure. Building and Environment 33, 115-133. https://doi.org/10.2148/benv.33.1.115

GUZMÁN RAMÍREZ, A. \& HERNÁNDEZ SAINZ, K. M. (2013). La fragmentación urbana y la segregación social una aproximación conceptual. Revista Legado de Arquitectura y Diseño, (14), 41-55. https://www.redalyc.org/articulo. oa?id=4779/477947373004

HALLER, A. (2017). Los impactos del crecimiento urbano en los campesinos andinos. Un estudio de percepción en la zona rural-urbana de Huancayo, Perú. Espacio y Desarrollo, 29, 37-56. doi.org/10.18800/espacioydesarrollo.201701.002

KLEEREKOPER, L.; VAN ESCH, M. \& SALCEDO, T. (2012). How to make a city climate-proof, addressing the urban heat island effect. Resources, Conservation and Recycling, 30-38. doi.org/10.1016/j.resconrec.2011.06.004

MAZA, J. A.; V. BURGOS; LÓPEZ, P. \& BENEGAS, V. (2004). Sustentabilidad hidrológica de urbanizaciones en piedemonte. Informe Técnico. Instituto Nacional del Agua - INA, Región Cuyo, Mendoza, Argentina. DOI: 10.13140/RG.2.1.2487.7289

MERTINS, G. (2000). Ciudades medianas en América Latina: criterios, indicadores y el intento de un modelo de su diferenciación socio-espacial y funcional. Espacio y Desarrollo, 12: 123-140. http://revistas.pucp.edu.pe/index.php/espacioydesarrollo/article/view/8081.

MIDDEL, A.; CHHETRI, N. \& QUAY, R. (2015). Urban forestry and cool roofs: Assessment of heat mitigation strategies in Phoenix residential neighborhoods. Urban Forestry \& Urban Greening, 14-1, 178-186. doi.org/10.1016/j. ufug.2014.09.010.

MIDDEL, A.; HÄB, K.; BRAZEL, A. J.; MARTIN, C. A. \& GUHATHAKURTA, S. (2014). Impact of urban form and design on mid-afternoon microclimate in Phoenix Local Climate Zones. Landscape and Urban Planning, 122, 16-28. doi.org/10.1016/j.landurbplan.2013.11.004 
MINISTERIO DE AMBIENTE Y DESARROLLO. Ley 4886/83 (1983). Disposición del suelo de zona oeste del gran Mendoza. Provincia de Mendoza. Argentina. http:// gxportal.mendoza.gov.ar

MOSCHELLA, P. (2017). Variación del paisaje para la gestión sostenible del territorio. In: Transversal: Acciones de integración en el territorio peruano (J. Stillemans, J. Canziani, M. Vilela \& P. Dam Mazzi, eds.): 333-346. Pontificia Universidad Católica del Perú.

NACIONES UNIDAS (2016). Resolución 71/256 de la Asamblea General "Nueva Agenda Urbana”, 23 de diciembre de 2016. https://undocs.org/es/A/RES/71/256.

NARUMI, D.; SHIGEMATSU, K. \& SHIMODA, Y. (2009). Effect of the Evaporative Cooling Techniques by Spraying Mist Water on Reducing Urban Heat Flux and Saving Energy in Apartment House. - 2nd Int. Conf. Countermeas. Osaka University Japan.

OKE, T. (2004). Initial guidance to obtain representative meteorological observations at urban sites. Geneva: IOM Report No. 81, World Meteorological Organization.

OLIVA, L. \& CASTRO, I. (2015). La década loteada. Unidiversidad, Datos interactivos, Ordenamiento territorial. Universidad Nacional de Cuyo. https://www. unidiversidad.com.ar/ladecadaloteada

PERINI, K. \& MAGLIOCCO, A. (2014). Effects of vegetation, urban density, building height, and atmospheric conditions on local temperatures and thermal comfort. Urban Forestry \& Urban Greening, 13, 495-506. doi.org/10.1016/j.ufug.2014.03.003

ROGERS, R. (2001). Ciudades para un pequeño planeta. Gustavo Gili, XI, 180 pp.

ROITMAN, S. (2003). Barrios cerrados y segregación social urbana. Scripta Nova. Revista Electrónica de Geografía y Ciencias Sociales. Universidad de Barcelona. 5, 146(118). http://www.ub.es/geocrit/sn/sn-146(118).htm

ROMERO, H. \& TOLEDO, X. (1998). Crecimiento económico y desigualdades regionales en Chile; indicadores de evaluación ambiental estratégica. Anales de la Sociedad Chilena de Ciencias Geográficas: 537-570.

SÁNCHEZ, M. (2015). Construir la ciudad desde los espacios públicos: herramientas de análisis y evaluación de tipologías urbanas para la integración socio-espacial. Revista de Urbanismo, (32), 127-138. doi:10.5354/0717-5051.2015.35459

SCHMIDT, M. (2006). The contribution of rainwater harvesting against global warming. London, UK: Technische Universität Berlin, IWA Publishing.

SECRETARÍA DE AMBIENTE Y DESARROLLO SUSTENTABLE (1991). Ley 5804: Expropiación de terrenos para conservación de sistema ecológico, Mendoza. Argentina. En Gobierno de Mendoza, http://gxportal.mendoza.gov.ar 
SECRETARÍA DE AMBIENTE Y DESARROLLO SUSTENTABLE (1995). Decreto 1077/95. Provincia de Mendoza. Argentina. En Gobierno de Mendoza, http://www. gobernac.mendoza.gov.ar

SOSA, M. B.; CORREA, E., \& CANTóN, M. A. (2020). Eficacia de estrategias de disminución del calentamiento urbano. Estudio para una ciudad de clima árido. Informes de la Construcción, 72(559), e352. doi.org/10.3989/ ic. 66662

STADEL, C. (2000). Ciudades medianas y aspectos de la sustentabilidad urbana en la región andina. Espacio y Desarrollo, 12: 25-43. http://revistas.pucp.edu.pe/index. php/espacioydesarrollo/article/view/8082

STOCCO, S.; CANTÓN, A. \& CORREA, E. (2018). Alternativas de diseño para mejorar el desempeño ambiental de plazas urbanas de Mendoza (Argentina). Evaluación mediante simulación con ENVI-met 3.1. Informes de la Construcción, 70(550): e253. doi.org/10.3989/ic.16.154

STOcco, S. (2016). Impacto de la morfología y materialidad de las plazas en la calidad energético-ambiental de ciudades emplazadas en zonas áridas. Universidad Tecnológica Nacional.

SZUPIANY, E. (2018). La ciudad fragmentada. Una lectura de sus diversas expresiones para la caracterización del modelo latinoamericano. Estudios Sociales Contemporáneos 19. https://bdigital.uncu.edu.ar/objetos_digitales/11569/ szupiany-laciudadfragmentada.pdf

TUMINI, I. (2012). El microclima urbano de los espacios abiertos. Estudio de casos en Madrid. Madrid, España: Universidad Politécnica de Madrid.

VILELA, M. \& MOSCHELLA, P. (2017). Paisaje y expansión urbana sobre espacios naturales en ciudades intermedias. El caso de Purrumpampa en Huamachuco, La Libertad, Perú. Bulletin de l'Institut français d'études andines, 46 (3). DOI: 10.4000/bifea.9003

VILLALOBOS, A. (2015). Historia urbana de Mendoza. Del siglo XVI al XXI. Suplemento Diseño urbano. Facultad de Ingeniería, Universidad Nacional de Cuyo.

WANG, Y. \& AKBARI, H. (2016). Analysis of urban heat island phenomenon and mitigation solutions evaluation for Montreal. Sustainable Cities and Society, 26: 438446. doi.org/10.1016/j.scs.2016.04.015

ZAPATERo SANTOS, M. A. (2017). Densidad urbana: Concepto y Metodología. Análisis Comparativo de los tejidos de Madrid. ETS Arquitectura (UPM). http:// oa.upm.es/45491/ 